\title{
SYNTHESIS OF 3 SUBSTITUTED ISOQUINOLIN-1-YL-2-(CYCLOALK-2-ENYLIDENE) HYDRAZINES AND THEIR ANTIMICROBIAL PROPERTIES
}

\author{
P. MANIVEL, S. MOHANA ROOPAN, R. SATHISH KUMAR, F. NAWAZ KHAN* \\ * Organic and Medicinal Chemistry Research Laboratory, Organic Chemistry Division, \\ School of Science and Humanities, \\ VIT-University, Vellore 632 014, Tamil Nadu, India. \\ (Received: September 3, 2008 - Accepted: December 4, 2008)
}

\begin{abstract}
New hydrazine derivatives were synthesized via reaction between 1, 3 cyclic diketones and hydrazinoisoquinoline derivatives. The reaction proceeded smoothly in ethanol under reflux temperature and furnished products in excellent yields (76-87\%). The products have been purified and fully characterized by spectroscopy techniques. The compounds 8a-c showed good bacterial inhibition against Bacillus cerus and 8d-f showed good antifungal activity against Candida albicans.
\end{abstract}

Key words: 1, 3-cyclic diketones, hydrazinoisoquinoline derivatives.

\section{INTRODUCTION}

Azomethine group bearing compounds are usually synthesized from the condensation of primary amines and active carbonyl groups. They are important class of compounds in medicinal and pharmaceutical field. They show biological applications including antibacterial, antifungal and antitumor activity $^{1-4}$. A variety of pharmacological effects are associated with isoquinoline derivatives including sedative, hypotensive, neuromuscular blocking and CNS activities $^{5}$. In recent years, there has been significant interest in the synthesis of these compounds and many approaches have been reported ${ }^{6,7}$. Although Schiff bases and azomethine derivatives are among the most thoroughly studied compounds, we were surprised that there has been no report of the isoquinolinylsubstituted azomethine derivatives. As part of a program to synthesize new heterocyclic compounds as potential pharmaceuticals ${ }^{8-15}$, we have investigated this reaction. We herein report the results of this study. The titled compounds were synthesized from homophthalic acid by five step synthesis. Homophthalic acid (1), on refluxing with acid chloride (2) in the absence of solvent yielded 3 -substituted isocoumarin ${ }^{8}(\mathbf{3})$. Compound $\mathbf{3}$ on reaction with ammonia in the presence of ethanol yielded 3-substituted isoquinolinone (4). Compound 4 in presence of $\mathrm{POCl}_{3}$ yielded 1-Chloro-3-substituted isoquinoline ${ }^{16}(\mathbf{5})$, which on reaction with hydrazine hydrate yielded 1-hydrazino-3-substituted isoquinoline ${ }^{17-19}(\mathbf{6})$. The titled compounds $(\mathbf{8})$ were prepared by treating $(\mathbf{6})$ with appropriate 1,3 diketones (7). The purity of synthesized compounds was monitored by thin layer chromatography (TLC) and LCMS elemental analyses and structures were identified by spectral data.

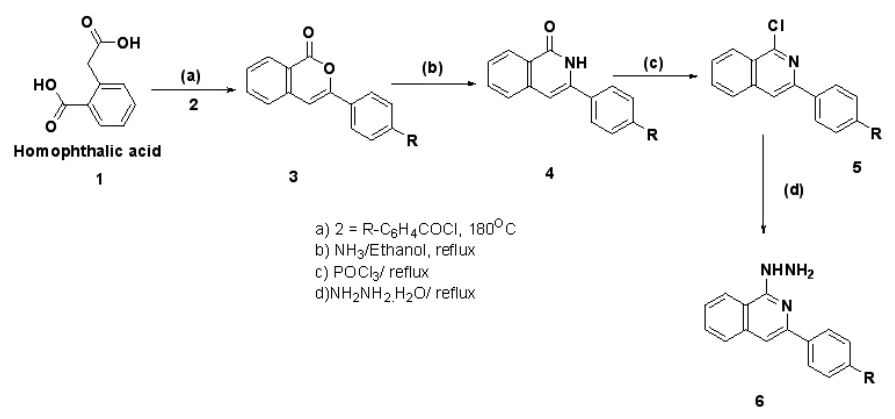<smiles>[R]c1ccc(-c2cc3ccccc3c(N)n2)cc1</smiles>

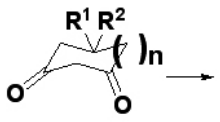

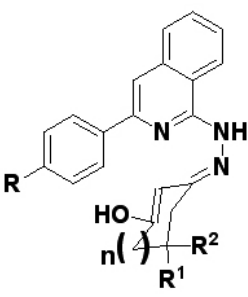

$6(a-b)$

$$
7(a-c)
$$

$n=0, R=H, R^{1}=H, R^{2}=H, \quad 8 a$ $n=1, R=H, R^{1}=H, R^{2}=H, \quad 8 b$ $\mathrm{n}=1, \mathrm{R}=\mathrm{H}, \mathrm{R}^{1}=\mathrm{CH}_{3}, \mathrm{R}^{2}=\mathrm{CH}_{3}, \mathbf{8 c}$ $\mathrm{n}=\mathrm{O}, \mathrm{R}=\mathrm{Cl}, \mathrm{R}^{1}=\mathrm{H}, \mathrm{R}^{2}=\mathrm{H}, \quad \mathbf{8 d}$ $n=1, R=C l, R^{1}=H, R^{2}=H, \quad 8 e$ $\mathrm{n}=1, \mathrm{R}=\mathrm{Cl}, \mathrm{R}^{1}=\mathrm{CH}_{3}, \mathrm{R}^{2}=\mathrm{CH}_{3}, \mathbf{8 f}$

Scheme: 1 Synthesis of Isoquinolin-1-yl-2-(cycloalk-2-enylidene) hydrazines

\section{EXPERIMENTAL SECTION}

Materials and Methods

Chemicals were purchased from Aldrich Chemical Co. and used as such without further purification. TLC was performed on silica plates with visualization by UV-light. Melting points were taken in open capillary tubes and corrected with reference to benzoic acid. IR spectra in $\mathrm{KBr}$ pellets were recorded on Nucon Infrared spectrophotometer. ${ }^{1} \mathrm{H}$ NMR $(400 \mathrm{MHz})$ and ${ }^{13} \mathrm{C}$ NMR (100 MHz) spectra were recorded on a Bruker $400 \mathrm{MHz}$ spectrometer in $\mathrm{CDCl}_{3}$ or DMSO (with TMS for ${ }^{1} \mathrm{H}$ NMR and DMSO for ${ }^{13} \mathrm{C}$ NMR as internal references). LC-MS analyses were performed with LCMS-Agilent- 1100 series Ion Trap. (8a-f)

Synthesis of Isoquinolin-1-yl-2-(cycloalk-2-enylidene) hydrazines

The hydrazine, (6a-b) and 1,3-diketones, (7a-c) were taken in ethanol (1:1 ratio) and refluxed under nitrogen atmosphere for overnight. Then reaction

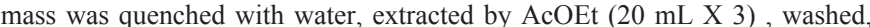
dried, concentrated and purified by column chromatography using silica gel to get the Schiff bases, (8a-f). The products obtained were characterized by IR, LCMS, ${ }^{1} \mathrm{H}-\mathrm{NMR}$ and ${ }^{13} \mathrm{C}-\mathrm{NMR}$ techniques. The reaction of hydrazine $\mathbf{6} \mathbf{a}-\mathbf{b}$ with various diketones, 7 a-c was tabulated (Table 1).

The spectral data of compounds, $\mathbf{8 a - 8} \mathbf{f}$ is given below.

(17Z)-2-(cyclo-3-hydroxy-pent-2-enylidene)-1-(3-phenylisoquinolin1-yl)hydrazine (8a) Dark brown solid, IR cm cm $^{-1} 3438(-\mathrm{OH}), 3326(-\mathrm{NH})$, $1714(\mathrm{C}=\mathrm{C}), 1649(\mathrm{C}=\mathrm{N}){ }^{1}{ }^{\mathrm{H}} \mathrm{NMR}\left(400 \mathrm{MHz}, \mathrm{DMSO}-\mathrm{d}_{6}\right) 9.73(\mathrm{~s}, 1 \mathrm{H},-\mathrm{OH})$, $9.51(\mathrm{~s}, 1 \mathrm{H}), 8,31(\mathrm{~s}, 1 \mathrm{H}), 8.25-8.23(\mathrm{~d}, \mathrm{~J}=8.0 \mathrm{~Hz}, 2 \mathrm{H}), 8.16-8.14(\mathrm{~d}, \mathrm{~J}=8.0 \mathrm{~Hz}$, $1 \mathrm{H}), 7.90-7.88(\mathrm{~d}, \mathrm{~J}=8.2 \mathrm{~Hz}, 1 \mathrm{H}), 7.78(\mathrm{~s}, 1 \mathrm{H},-\mathrm{NH}), 7.72-7.68$ (t, J=7.5 Hz, $1 \mathrm{H}), 7.57-7.53(\mathrm{t}, \mathrm{J}=4.0 \mathrm{~Hz}, 2 \mathrm{H}), 7.47-7.44(\mathrm{~m}, 1 \mathrm{H}), 7.38-7.34(\mathrm{t}, \mathrm{J}=7.2 \mathrm{~Hz}$, $1 \mathrm{H}), 4.84(\mathrm{~s}, 1 \mathrm{H}, \mathrm{C}=\mathrm{C} H), 2.74-2.66\left(\mathrm{~m}, 2 \mathrm{H}, \mathrm{C}-\mathrm{CH}_{2}\right), 2.28\left(\mathrm{~m}, 2 \mathrm{H}, \mathrm{C}-\mathrm{CH}_{2}\right)$; 
${ }^{13} \mathrm{C}$ NMR (100 MHz, DMSO-d $) 202.35(\mathrm{C}=\mathrm{COH}), 154.01(\mathrm{C}=\mathrm{C}-\mathrm{NH}), 147.84$ $(\mathrm{C}-\mathrm{C}=\mathrm{N}), 139.46,138.20,133.46,130.90,128.97,2 \mathrm{X}(128.79), 2 \mathrm{X}(127.83)$ $126.71,123.17,116.51,108.67$ (aromatic carbons), 99.35, 79.63, 34.15, 25.76 (alicyclic carbons); LCMS: m/e 316.12; Mol. Formula $\mathrm{C}_{20} \mathrm{H}_{17} \mathrm{~N}_{3} \mathrm{O}$, Mol. Wt.: 315.37 .

Table 1.- Synthesis of Isoquinolin-1-yl-2-(cycloalk-2-enylidene) hydrazines from 1, 3 cyclic diketones and hydrazinoisoquinoline derivatives.

\begin{tabular}{|c|c|c|c|c|c|c|c|}
\hline Entry & Compounds $^{\mathbf{a}}$ & $\mathbf{R}$ & $\mathbf{n}$ & $\mathbf{R}^{1}$ & $\mathbf{R}^{2}$ & $\boldsymbol{m}^{\circ} \mathbf{C}$ & $\begin{array}{c}\text { bYield } \\
\mathbf{\%}\end{array}$ \\
\hline 1 & $8 \mathrm{a}$ & $\mathrm{H}$ & 0 & $\mathrm{H}$ & $\mathrm{H}$ & $\begin{array}{c}201-203 \\
{ }^{\circ} \mathrm{C}\end{array}$ & 80 \\
\hline 2 & $8 \mathrm{~b}$ & $\mathrm{H}$ & 1 & $\mathrm{H}$ & $\mathrm{H}$ & $\begin{array}{c}191-193 \\
{ }^{\circ} \mathrm{C}\end{array}$ & 82 \\
\hline 3 & $8 \mathrm{c}$ & $\mathrm{H}$ & 1 & $\mathrm{CH}_{3}$ & $\mathrm{CH}_{3}$ & $\begin{array}{c}205-207 \\
{ }^{\circ} \mathrm{C}\end{array}$ & 85 \\
\hline 4 & $8 \mathrm{~d}$ & $\mathrm{Cl}$ & 0 & $\mathrm{H}$ & $\mathrm{H}$ & $\begin{array}{c}235-237 \\
{ }^{\circ} \mathrm{C}\end{array}$ & 76 \\
\hline 5 & $8 \mathrm{e}$ & $\mathrm{Cl}$ & 1 & $\mathrm{H}$ & $\mathrm{H}$ & $\begin{array}{c}193- \\
195^{\circ} \mathrm{C}\end{array}$ & 83 \\
\hline 6 & $8 \mathrm{f}$ & $\mathrm{Cl}$ & 1 & $\mathrm{CH}_{3}$ & $\mathrm{CH}_{3}$ & $\begin{array}{c}215- \\
217^{\circ} \mathrm{C}\end{array}$ & 87 \\
\hline
\end{tabular}

${ }^{a}$ All products were identified by ${ }^{1} \mathrm{H},{ }^{13} \mathrm{C} \mathrm{NMR}$, bisolated yields.

(17Z)-2-(cyclo-3-hydroxy-hex-2-enylidene)-1-(3-phenylisoquinolin1-yl)hydrazine (8b) Brown solid, IR cm ${ }^{-1} 3324(-\mathrm{OH}), 3212(-\mathrm{NH}), 1613$ $(\mathrm{C}=\mathrm{N}), 1583(\mathrm{C}=\mathrm{C}) ;{ }^{1} \mathrm{H}$ NMR $(400 \mathrm{MHz}, \mathrm{DMSO}-\mathrm{d}) 9.55(\mathrm{~s}, 1 \mathrm{H},-\mathrm{OH}), 9.01$ $(\mathrm{s}, 1 \mathrm{H}), 8.28-8.26(\mathrm{~d}, \mathrm{~J}=8.3 \mathrm{~Hz}, 1 \mathrm{H}), 8.17-8.15(\mathrm{~d}, \mathrm{~J}=8.2 \mathrm{~Hz}, 2 \mathrm{H}), 7.89-7.87$ $(\mathrm{d}, \mathrm{J}=8.0 \mathrm{~Hz}, 1 \mathrm{H}), 7.77(\mathrm{~s}, 1 \mathrm{H},-\mathrm{NH}), 7.72-7.68(\mathrm{t}, \mathrm{J}=7.5 \mathrm{~Hz}, 1 \mathrm{H}), 7.57-7.53$ $(\mathrm{t}, \mathrm{J}=4.0 \mathrm{~Hz}, 1 \mathrm{H}), 7.48-7.44(\mathrm{~m}, 2 \mathrm{H}), 7.38-7.34(\mathrm{t}, \mathrm{J}=7.2 \mathrm{~Hz}, 1 \mathrm{H}), 5.01(\mathrm{~s}, 1 \mathrm{H}$ $\mathrm{C}=\mathrm{CH}), 2.55\left(\mathrm{~m}, 2 \mathrm{H}, \mathrm{C}-\mathrm{CH}_{2}\right), 2.14-2.11\left(\mathrm{~m}, 2 \mathrm{H}, \mathrm{C}-\mathrm{C} H_{2}\right), 1.94-1.93(\mathrm{~d}, 2 \mathrm{H}$, $\left.\mathrm{C}-\mathrm{CH}_{2}\right) ;{ }^{13} \mathrm{C}$ NMR $\left(100 \mathrm{MHz}, \mathrm{DMSO}-\mathrm{d}_{6}\right) 195.35(\mathrm{C}=\mathrm{COH}), 164.76,154.01$, $147.86(\mathrm{C}-C=\mathrm{N}), 139.53,138.25,130.86,128.96,2 \mathrm{X}(128.79), 2 \mathrm{X}(127.87)$, $126.69,123.09,116.49,108.45$ (aromatic carbons), 96.41, 79.63, 37.22, 26.63, 22.34 (alicyclic carbons); LCMS: m/e 330.1; Mol. Formula $\mathrm{C}_{21} \mathrm{H}_{19} \mathrm{~N}_{3} \mathrm{O}$, Mol. Wt.: 329.4 .

(17Z)-2-(5,5-dimethylcyclo-3-hydroxy-hex-2-enylidene)-1-(3phenylisoquinolin-1-yl)hydrazine (8c) Pale yellow, IR $\mathrm{cm}^{-1}$ 3217(-OH), 3032(-NH), 1625(C=N), $1593(\mathrm{C}=\mathrm{C}) ;{ }^{1} \mathrm{H}$ NMR $\left(400 \mathrm{MHz}, \mathrm{DMSO}-\mathrm{d}_{\mathrm{f}}\right) 9.55(\mathrm{~s}$, $1 \mathrm{H},-\mathrm{OH}), 8.99(\mathrm{~s}, 1 \mathrm{H}), 8.29-8.27(\mathrm{~d}, \mathrm{~J}=8.3 \mathrm{~Hz}, 1 \mathrm{H}), 8.19-8.17$ (t, J=4.2 Hz, $2 \mathrm{H}), 7.89-7.87(\mathrm{~d}, \mathrm{~J}=8.0 \mathrm{~Hz}, 1 \mathrm{H}), 7.78(\mathrm{~s}, 1 \mathrm{H},-\mathrm{NH}), 7.71-7.68(\mathrm{t}, \mathrm{J}=7 . .5$ $\mathrm{Hz}, 1 \mathrm{H}), 7.56-7.52(\mathrm{~m}, 1 \mathrm{H}), 7.44-7.41(\mathrm{~m}, 2 \mathrm{H}), 7.36-7.33(\mathrm{t}, \mathrm{J}=7.2 \mathrm{~Hz}, 1 \mathrm{H})$ $4.98(\mathrm{~s}, 1 \mathrm{H}, \mathrm{C}=\mathrm{CH}), 2.42\left(\mathrm{~s}, 2 \mathrm{H}, \mathrm{C}-\mathrm{CH}_{2}\right), 2.01(\mathrm{~s}, 2 \mathrm{H}, \mathrm{C}-\mathrm{CH}), 1.07(\mathrm{~s}, 6 \mathrm{H}$, $\left.-\mathrm{CH}_{3}\right) ;{ }^{13} \mathrm{C}$ NMR $(100 \mathrm{MHz}, \mathrm{DMSO}-\mathrm{d})$ 194.91 $(\mathrm{C}=\mathrm{COH}), 164.76(\mathrm{C}=\mathrm{C}-\mathrm{NH})$, 154.07 $\left(\mathrm{CH}_{2}-C=\mathrm{N}\right), 147.83(-C=\mathrm{N}), 133.42,139.39,138.22,130.84,128.83,2$ $\mathrm{X}(128.78), 2 \mathrm{X}(127.86), 126.71,123.07,116.47,108.39$ (aromatic carbons), $94.83,79.67,51.08,33.10$ (alicyclic carbons), 2 X $28.54\left(-\mathrm{CH}_{3}\right) ; \mathrm{LCMS}: \mathrm{m} / \mathrm{e}$ 358.1; Mol. Formula $\mathrm{C}_{23} \mathrm{H}_{23} \mathrm{~N}_{3} \mathrm{O}$, Mol. Wt.: 357.45 .

(18Z)-1-(3-(4-chlorophenyl)isoquinolin-1-yl)-2-(3-hydroxy-cyclopent-2-enylidene)hydrazine (8d) Pale yellow, IR $\mathrm{cm}^{-1} 3428(-\mathrm{OH}), 3349$ $(-\mathrm{NH}), 1705(\mathrm{C}=\mathrm{N}), 1651(\mathrm{C}=\mathrm{C}) ;{ }^{1} \mathrm{H}$ NMR $(400 \mathrm{MHz}, \quad \mathrm{DMSO}-\mathrm{d}) 9.77(\mathrm{~s}$, $1 \mathrm{H},-\mathrm{OH}), 9.52(\mathrm{~s}, 1 \mathrm{H}), 8.25-8.23(\mathrm{~d}, \mathrm{~J}=8.3 \mathrm{~Hz}, 1 \mathrm{H}), 8.18-8.16$ (d, J=8.5 Hz, $2 \mathrm{H}), 7.89-7.87(\mathrm{~d}, \mathrm{~J}=8.1 \mathrm{~Hz}, 1 \mathrm{H}), 7.81(\mathrm{~s}, 1 \mathrm{H},-\mathrm{NH}), 7.73-7.69(\mathrm{t}, \mathrm{J}=7.5 \mathrm{~Hz}$, $1 \mathrm{H}), 7.58-7.51(\mathrm{~m}, 3 \mathrm{H})), 4.83(\mathrm{~s}, 1 \mathrm{H},=\mathrm{CH}), 2.75\left(\mathrm{~s}, 2 \mathrm{H},-\mathrm{CH}_{2}\right), 2.29(\mathrm{~s}, 2 \mathrm{H}$, $\left.-\mathrm{CH}_{2}\right) ;{ }^{13} \mathrm{C}$ NMR $(100 \mathrm{MHz}$, DMSO-d $) 202.44(\mathrm{C}=\mathrm{COH}), 178.57(\mathrm{C}=\mathrm{C}-\mathrm{NH})$, $154.05\left(\mathrm{CH}_{2}-C=\mathrm{N}\right), 146.59(-C=\mathrm{N}), 138.36,138.12,133.46,131.00,128.99,2$ $\mathrm{X}(128.37), 2 \mathrm{X}(127.90), 126.95,123.19,116.63,108.85$ (aromatic carbons), 99.36, 34.15, 25.75(alicyclic carbons); LCMS: m/e 350.0; Mol. Formula $\mathrm{C}_{20} \mathrm{H}_{16} \mathrm{ClN}_{3} \mathrm{O}$, Mol. Wt.: 349.81 .

(18Z)-1-(3-(4-chlorophenyl)isoquinolin-1-yl)-2-(-3-hydroxy-cyclohex2-enylidene)hydrazine (8e) Pale yellow, IR cm-1 3331(-OH), $3230(-\mathrm{NH})$, 1685(C=N), $1584(\mathrm{C}=\mathrm{C}) ;{ }^{1} \mathrm{H}$ NMR (400 MHz, DMSO-d $) 9.58(\mathrm{~s}, 1 \mathrm{H},-\mathrm{OH})$, $9.00(\mathrm{~s}, 1 \mathrm{H}), 8.28-8.26(\mathrm{~d}, \mathrm{~J}=8.3 \mathrm{~Hz}, 1 \mathrm{H}), 8.18-8.16(\mathrm{~d}, \mathrm{~J}=8.6 \mathrm{~Hz}, 2 \mathrm{H}), 7.89-$ $7.87(\mathrm{~d}, \mathrm{~J}=8.1 \mathrm{~Hz}, 1 \mathrm{H}), 7.80(\mathrm{~s}, 1 \mathrm{H},-\mathrm{NH}), 7.72-7.69(\mathrm{t}, \mathrm{J}=7.5 \mathrm{~Hz}, 1 \mathrm{H}), 7.58$ $7.51(\mathrm{~m}, 3 \mathrm{H}), 4.99(\mathrm{~s}, 1 \mathrm{H}, \mathrm{C}=\mathrm{CH}), 2.55\left(\mathrm{t}, 2 \mathrm{H},-\mathrm{CH}_{2}\right), 2,14-2.11(\mathrm{~m}, 2 \mathrm{H}$, $\left.-\mathrm{CH}_{2}\right), 1.92\left(\mathrm{t}, 2 \mathrm{H},-\mathrm{CH}_{2}\right) ;{ }^{13} \mathrm{C}$ NMR $(100 \mathrm{MHz}$, DMSO-d $) 195.39(\mathrm{C}=\mathrm{COH})$, $146.59(\mathrm{C}=C-\mathrm{NH}), 164.76,154.01,138.41,138.13,133.44,130.98,128.97,2$ $\mathrm{X}(128.35), 2 \mathrm{X}$ (127.92), 126.93, 123.10, 116.58, 108.65 (aromatic carbons), $96.41,79.63,37.22,22.34$ (alicyclic carbons); LCMS: m/e 364.0; Mol. Formula $\mathrm{C}_{21} \mathrm{H}_{18} \mathrm{ClN}_{3} \mathrm{O}$, Mol. Wt.: 363.84 .
(18Z)-1-(3-(4-chlorophenyl)isoquinolin-1-yl)-2-(3-hydroxy-5,5dimethylcyclohex-2-enylidene) hydrazine ( 8 f ) Pale yellow, IR $\mathrm{cm}^{-1} 3210$ $(-\mathrm{OH}), 3027$ (-NH str.), $1626(\mathrm{C}=\mathrm{N}), 1594(\mathrm{C}=\mathrm{C}) ;{ }^{1} \mathrm{H}$ NMR $(400 \mathrm{MHz}$, DMSO-d ) 9.59 (s, 1H, -OH), 8.99 (s, 1H), $8.31-8.27$ (t, J=7.3 Hz, 1H), 8.22$8.18(\mathrm{~m}, 2 \mathrm{H}), 7.89-7.87(\mathrm{~d}, \mathrm{~J}=8.0 \mathrm{~Hz}, 1 \mathrm{H}), 7.81(\mathrm{~s}, 1 \mathrm{H},-\mathrm{NH}), 7.73-7.69$ $(\mathrm{m}, 1 \mathrm{H}), 7.58-7.54(\mathrm{~m}, 1 \mathrm{H}), 7.51-7.48(\mathrm{~m}, 2 \mathrm{H}), 4.96(\mathrm{~s}, 1 \mathrm{H},=\mathrm{CH}), 2.42$ $\left(\mathrm{s}, 2 \mathrm{H},-\mathrm{CH}_{2}\right), 2,01\left(\mathrm{~s}, 2 \mathrm{H},-\mathrm{CH}_{2}\right), 1.07\left(\mathrm{~s}, 6 \mathrm{H},-\mathrm{CH}_{3}\right) ;{ }^{13} \mathrm{C}$ NMR $(100 \mathrm{MHz}$, DMSO-d $) 194.96(\mathrm{C}=C \mathrm{OH}), 164.71(\mathrm{C}=C-\mathrm{NH}), 154.13\left(\mathrm{CH}_{2}-\mathrm{C}=\mathrm{N}\right), 146.54(-$ $C=\mathrm{N}), 138.28,138.13,133.44,130.98,128.85,2$ X (128.38), 2 X (127.93), $126.95,123.10,116.57,108.62$ (aromatic carbons), 94.82, 79.63, 51.07,

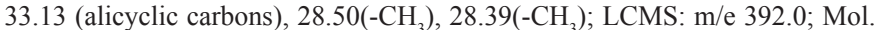
Formula $\mathrm{C}_{23} \mathrm{H}_{22} \mathrm{ClN}_{3} \mathrm{O}$, Mol. Wt.: 391.89 .

Antimicrobial properties

Cultures of bacteria were grown on nutrient broth (HiMedia) at $37^{\circ} \mathrm{C}$ for $12-14 \mathrm{hr}$ and that of fungus on Sabouraud dextrose broth (HiMedia) at $28^{\circ} \mathrm{C}$ for $48 \mathrm{hr}$ and were maintained on respective agar slants at $4^{\circ} \mathrm{C}$. The compounds 8a-f were screened for their antibacterial activities against Escherichia coli, Salmonella typhi, Proteus mirabilis, Bacillus cerus, Staphylococcus aureus, and antifungal activities against Candida albicans, Aspergillus flavus, Aspergillus niger by agar well technique ${ }^{20}$. Standard antibacterial eftazidime, Chloramphenicol and antifungal Nalidixic acid were also tested under similar conditions for comparison. The compounds 8a-f of $4 \mathrm{mg} / \mathrm{ml}$ concentration was used as stock solution, from this $100 \mu \mathrm{l}$ was loaded to each well. The antimicrobial properties were duplicated and the averages were taken.

\section{RESULTS AND DISCUSSION}

The 1-hydrazino-3-substituted isoquinoline (6a-b) required for our reaction were prepared from isocoumarin ${ }^{8,11}$ by following reported procedures ${ }^{16-18}$. Purified 1-hydrazino-3-substituted isoquinoline derivatives were allowed to react with 1,3-diketones (7a-c) in presence of anhydrous ethanol under nitrogen atmosphere to give the corresponding Isoquinolin-1-yl-2-(cycloalk2-enylidene)hydrazines derivatives (8a-f), (Scheme 1; Table 1). The reaction producing hydrazine derivatives by a simple and an efficient route gave good yields. Products of the reaction have been isolated, purified and characterized by various spectral techniques such as IR, LC-MS, ${ }^{1} \mathrm{H}-\mathrm{NMR}$ and ${ }^{13} \mathrm{C}-\mathrm{NMR}$ techniques.

Table 2 Antibacterial and Antifungal Activities of Compounds 8a-f

\begin{tabular}{|c|c|c|c|c|c|c|c|}
\hline \multirow[b]{2}{*}{ Organisms } & \multicolumn{6}{|c|}{ Titled compounds } & \multirow{2}{*}{$\begin{array}{c}\text { Standards } \\
\text { (30mcg/disc) }\end{array}$} \\
\hline & $8 a$ & $8 b$ & $8 \mathrm{c}$ & $8 d$ & $8 \mathrm{e}$ & $8 f$ & \\
\hline \multicolumn{7}{|c|}{ Antibacterial activity } & \\
\hline E. coli & - & - & - & - & - & - & $23\left(\mathrm{Ca}^{30}\right)$ \\
\hline S. typhi & - & - & - & - & - & - & $28\left(C^{30}\right)$ \\
\hline P. mirabilis & - & - & - & - & - & - & $20\left(\mathrm{Ca}^{30}\right)$ \\
\hline B. cerus & + & + & + & - & - & - & $20\left(\mathrm{Ca}^{30}\right)$ \\
\hline S. aureus & - & - & - & - & - & - & $22\left(\mathrm{Na}^{30}\right)$ \\
\hline \multicolumn{7}{|c|}{ Antifungal activity } & \\
\hline C. albicans & - & - & - & ++ & ++ & ++ & $19\left(\mathrm{Na}^{30}\right)$ \\
\hline A. flavus & - & - & - & - & - & - & - \\
\hline A. niger & - & - & - & - & - & - & - \\
\hline
\end{tabular}

$\mathrm{Ca}^{30}$ - Ceftazidime, $\mathrm{C}^{30}$ - Chloramphenicol, $\mathbf{N a}^{30}$ - Nalidixic acid, Control $=$ DMSO,$+=12$ to $13 \mathrm{~mm},++=14$ to $15 \mathrm{~mm}$

\section{LCMS spectra}

The LCMS of titled compounds showed a molecular ion peak M+ in the positive mode. The molecular ion peak for $8 \mathbf{a}$ was observed at $\mathrm{m} / \mathrm{z}=316.12$. This is also supported by the LCMS analysis of other compounds, $8 \mathbf{b - 8 f}$. 


\section{IR and NMR spectra}

In IR spectrum, reactant diketones 7a-c gave peaks at around 1730-1710 $\mathrm{cm}^{-1}$ indicates the presence of carbonyl groups where as in products 8a-f obtained from 7a-c gave peaks at around $3300-3200 \mathrm{~cm}^{-1}$ indicates the reaction between $7 \mathrm{a}-\mathrm{c}$ and $6 \mathrm{a}-\mathrm{f}$ with the formation of an enolic group from an unreacted keto group of diketones.

In ${ }^{1} \mathrm{H}$ NMR spectra, peaks at $\delta 9.55-9.75 \mathrm{ppm}$ indicates the presence of an enolic hydroxyl group. Similarly ${ }^{13} \mathrm{C}$ NMR peaks at around $\delta 190-205 \mathrm{ppm}$ indicates enolic carbon thereby confirming isomerism of an unreacted keto group of diketone to enol form.

Antimicrobial studies

Among various Isoquinolin-1-yl-2-(cycloalk-2-enylidene)hydrazines, those with electron rich groups present in the compounds (8a-c) posses good antibacterial activity against B.cerus as well as those having electron deficient groups present in the compounds (8d-f) posses good antifungal activity against $C$. albicans. From the screening data given in Table 2, it is evident that compounds $\mathbf{8 a}, \mathbf{8 b}$ and $\mathbf{8 c}$ exhibited the highest degree of inhibition only against bacterial species $B$. cerus, compounds $8 \mathbf{d}, \mathbf{8 e}$ and $\mathbf{8 f}$ showed highest degree of inhibition only against fungal species C. Albicans. However, the activities of tested compounds are less than that of the standard agent used.

\section{CONCLUSIONS}

We have described a simple method for the synthesis of new heterocyclic Schiff bases by using 1, 3 cyclic diketones and hydrazinoisoquinoline derivatives. The method offers several advantages including high yields and simple work up procedure for the transformation of 1-hydrazino-3-substituted isoquinoline into isoquinolin-1-yl-2-(cycloalk-2-enyldene) hydrazine. The antimicrobial activities including antibacterial and antifungal properties of the synthesized compounds showed the titled compounds as biologically valuable materials.

\section{ACKNOWLEDGEMENT}

Authors are thankful to VIT University, Vellore for providing research facilities. The authors wish to express their gratitude to SAIF, Indian Institute of Science, Bangalore; Spic Science foundation, Tuticorin and Syngene International Limited, Bangalore for their support of NMR, LCMS.

\section{REFERENCES}

1. K. Singh, M.S. Barwa, P. Tyagi, Eur. J. Med. Chem. 41, 1, (2006).

2. S.N. Pandeya, D. Sriram, G. Nath, E. Declercq, Eur. J. Pharmacol. 9, $25,(1999)$.

3. R. Mladenova, M. Ignatova, N. Manolova, T. Petrova, I. Rashkov, Eur. Polym. J. 38, 989, (2002).

4. O.M. Walsh, M.J. Meegan, R.M. Prendergast, T.A. Nakib, Eur. J. Med. Chem. 31, 989, (1996).

5. A. Padwa, R. Hennig, C.O. Kappe, T.S. Reger, J. Org. Chem. 63, 1144, (1998).

6. E. Garcia, S. Arrasate, E. Lete, N. Sotomayor, J. Org. Chem. 70, 10368 , (2005).

7. B.E. Maryanoff, H.C. Zhang, J.H. Cohen, I.J. Turchi, C.A. Maryanoff, Chem. Rev. 104, 1431, (2004).

8. S. Syed Tajudeen, F. Nawaz Khan, Synth.Commun. 37, 3649, (2007).

9. P. Manivel, S. Mohana Roopan, F. Nawaz Khan, J. Chil. Chem. Soc. 53 1609, (2008).

10. S. Mohana Roopan, T. Maiyalagan, F. Nawaz Khan, Can. J. chem. 86, 1019, (2008)

11. V.R. Hathwar, P. Manivel, F. Nawaz Khan, T.N. Guru Row, Acta Cryst E63, 03707, (2007).

12. V.R. Hathwar, P. Manivel, F. Nawaz Khan, T.N. Guru Row, Acta Cryst. E63, 03708, (2007).

13. V.R. Hathwar, P. Manivel, F. Nawaz Khan, T.N. Guru Row, Cryst. Eng. Comm. 11, 284, (2009)

14. V.R. Hathwar, K. Prabakaran, R. Subashini, P. Manivel, F. Nawaz Khan, Acta Cryst., E64, o2295, (2008)

15. P. Manivel, S. Mohana Roopan, D. Prem, F. Nawaz Khan, Phosphorus, Sulphur and Silicon and other related elements, (2009). In Press.

16. B.F. Powell, C.G. Overberger, J.P. Anselme, J. Het. chem. 20, 121, (1983).

17. A.A. Alhaider, M. Altef Abdel kader, E.J. Lien, J. Med. Chem. 28, 1394, (1983).

18. O. Charles, Okafor, J. Org. Chem. 47, 592, (1982).

19. M.M. Ismail, M. Abass, M.M. Hassan, Molecules 5, 1224, (2000).

20. G. Grover, S.G. Kini, Eur. J. Med. Chem. 41, 256, (2006). 